\title{
High brightness fully coherent $x$-ray amplifier seeded by a free-electron laser oscillator
}

\author{
Kai Li, ${ }^{1,2}$ Jiawei Yan, ${ }^{1,2}$ Chao Feng, ${ }^{1}$ Meng Zhang, ${ }^{1}$ and Haixiao Deng ${ }^{1, *}$ \\ ${ }^{1}$ Shanghai Institute of Applied Physics, Chinese Academy of Sciences, Shanghai 201800, China \\ ${ }^{2}$ University of Chinese Academy of Sciences, Beijing 100049, China
}

(Received 3 November 2017; published 17 April 2018)

\begin{abstract}
X-ray free-electron laser oscillator (XFELO) is expected to be a cutting-edge tool for fully coherent X-ray laser generation, and undulator taper technique is well-known for considerably increasing the efficiency of free-electron lasers (FELs). In order to combine the advantages of these two schemes, FEL amplifier seeded by XFELO is proposed by simply using a chirped electron beam. With the right choice of the beam parameters, the bunch tail is within the gain bandwidth of XFELO, and lase to saturation, which will be served as a seeding for further amplification. Meanwhile, the bunch head which is outside the gain bandwidth of XFELO, is preserved and used in the following FEL amplifier. It is found that the natural "double-horn" beam current, as well as residual energy chirp from chicane compressor, are quite suitable for the new scheme. Inheriting the advantages from XFELO seeding and undulator tapering, it is feasible to generate nearly terawatt level, fully coherent x-ray pulses with unprecedented shot-to-shot stability, which might open up new scientific opportunities in various research fields.
\end{abstract}

DOI: 10.1103/PhysRevAccelBeams.21.040702

\section{INTRODUCTION}

The successful operation of self-amplified spontaneous emission (SASE) [1,2] x-ray free-electron lasers (XFEL) [3-6] around the world have offered innovative approach for studying various areas such as atomic, molecular, and optical physics; condensed matter physics; mater in extreme conditions; chemistry and soft matter, and biology [7]. Starting from initial electron beam density shot noise, SASE is able to produce spatially coherent, tens of gravitational waves $(\mathrm{GW})$ and around $100 \mathrm{fs}$ temporal duration radiation pulses in short wavelength regime. However, its poor longitudinal coherence is characterized by tens to hundreds of spikes with chaotic phase relation. Improving the longitudinal coherence of XFEL is of great interests ever since the first light of Linac Coherent Light Source. Modifications to SASE, e.g., self-seeding [8,9] and slippage-boosted methods [10-12], have been proposed to achieve this goal. In Self-seeding scheme, a monochromator is inserted in the middle of undulator segments to spectral purify SASE and obtain a coherent signal which is then amplified in the downstream undulators. Although it has been successfully demonstrated to improve longitudinal

*denghaixiao@sinap.ac.cn

Published by the American Physical Society under the terms of the Creative Commons Attribution 4.0 International license. Further distribution of this work must maintain attribution to the author(s) and the published article's title, journal citation, and DOI. coherence experimentally, the intrinsic fluctuation of final output radiation inherited from SASE seed is a limitation for some applications. Other methods like speeding up the slippage in undulators will also suffer from large shot-toshot fluctuations. In addition, the preservation of temporal coherence and operation stability for external-seeding technique are still not clear in hard x-ray region [13-15].

Another major goal of XFEL development is to further enhance peak power and photon flux for increasing the resolution of x-ray imaging experiments [16,17]. In FEL process, sustainable energy extraction from electron beam is guaranteed by the resonant condition which ensures radiation field slips ahead one wavelength when electron beam propagates one undulator period. Saturation occurs for normal planar undulator when electrons lose too much energy and drop out of resonance with radiation wave. To preserve the resonant condition so that further increase FEL peak power to TW level, undulator parameters is precisely optimized to compensate the electron beam energy loss [18]. Based on the idea of variable parameter undulators, efforts have been continuously made to improve the energy extraction efficiency in the last decades [19,20]. Although the tapering technique has proved to be feasible in long wavelength regions such as microwave radiation and visible light regime [21,22]. Significantly enhancing XFEL efficiency is still challenging, due to the predicted sensitivity of tapered FEL to shot-to-shot power fluctuation and poor temporal coherence of SASE $[23,24]$.

Although the fully coherent and stable seed is favorable for the tapered amplifier to generate ultrahigh brightness 
$\mathrm{X}$-ray beam, the shortcomings of previous methods for enhancing SASE longitudinal coherence are the obstacles to the practical implementation of the tapering technique. An alternative robust $\mathrm{x}$-ray source which serves as a seed is crucial to further improve the XFEL performances. According to the conventional oscillator-amplifier configuration in laser community, it is straightforward to take x-ray FEL oscillator (XFELO) output as a seed signal for following FEL amplifier [25]. Utilizing relativistic electron beam as gain medium and crystal as optical cavity mirrors, XFELO starts from spontaneous radiation and its spectra is purified during each Bragg reflection. It is capable of generating stable, high repetition rate, fully coherent $\mathrm{x}$-ray pulses [26,27]. However, it is well-recognized that the performance of oscillator-amplifier FEL configuration will be limited by the electron beam over-bunching and energy spread growth induced by FEL modulation in the oscillator. In this paper, a novel and simple FEL amplifier seeded by XFELO is proposed by using a chirped electron beam. In this scheme, with the careful selection of electron beam chirp, only the tail electrons interact resonantly with radiation inside the XFELO. The electron beam then passes through a chicane delay, which makes the well-preserved head electrons overlap with seed laser from XFELO. More energy is drawn from the electron beam head in the following tapered FEL amplifier, thus substantially increases the photon flux. The coherent seeds from oscillator ensure high efficiency of tapering technique, and according to the simulation results, nearly terawatt level, ultra-high brightness, pretty stable x-ray pulses are delivered as an ideal light source for scientific users.

Using the parameters of Shanghai Coherent Light Facility (SCLF) as an example [28], we show that XFELO seeded amplifier is of potential for future XFEL facilities to significantly enhance FEL peak power, spectral brightness as well as output power stability. This paper is organized as following: the principles of our scheme is presented in Sec. II. The simulation results at $5 \mathrm{keV}$ photon energy using a $3 \mathrm{kA}$ flat-top electron beam is illustrated in Sec. III. The start-to-end simulations with SCLF parameters are discussed in Sec. IV and the output power stability analysis is shown in Sec. V. Finally, a brief conclusion is given in Sec. VI.

\section{THE PRINCIPLES DESCRIPTION}

Unlike conventional lasers whose wavelength is limited by the energy levels of gain materials, XFELO extracts power from the relativistic electron beam, thus the generation of photon energy is unbounded by gain medium theoretically. Taking advantages of high Bragg reflection crystals demonstrated experimentally recently [29], the $\mathrm{x}$-ray beam is trapped inside the resonator and oscillates back and forth. During each round trip, x-ray pulse interacts with an electron beam and obtains FEL gain inside the undulator. In order to ensure the growth of light power, undulator should be long enough to maintain sufficient single pass gain, i.e.

$$
R(1+G)>1,
$$

where $R$ is the total reflectivity of the crystal mirrors, and $G$ is the single pass gain. The $\mathrm{x}$-ray radiation is amplified during each round trip and grows exponentially until equilibrium. In the saturation regime, FEL gain degradation occurs due to the over-modulation of electron bunch induced by the high power x-ray. When the single pass gain equals to output coupling and round trip loss, the XFELO reaches equilibrium which produces stable output $\mathrm{X}$-ray pulses.

Similar to traditional laser, with carefully optimized electron beam quality, undulator length and cavity output coupling, XFELO is able to deliver stable, high repetition rate, fully coherent $\mathrm{X}$-ray pulses [30]. The peak power is typically several megawatt (MW) for low peak current electron beam $(\sim 10 \mathrm{~A})$ in the early XFELO proposals $[26,27,30,31]$,

and due to the narrow spectral acceptance of the Bragg crystal [32], typically tens of meV, the average brightness for XFELO output is predicted to be 2 orders of magnitudes higher than SASE. With the development of superconducting accelerator technology, several kA peak current Linac-based high repetition rate x-ray FELs are under construction $[33,34]$. The generated high brightness electron bunches is also suitable for high current XFELO operation. For a typical FEL oscillator, the maximum fractional energy extracted from electron beam is inverse proportion to $1 / N_{u}$, with $N_{u}$ is the number of undulator periods. For a given single pass gain, high peak current means short undulator length, which is expected to generate x-ray pulse with peak power nearly GW level. For further enhancing the peak power, Fig. 1 shows the schematic of XFELO seeded tapered FEL amplifier. A chicane is applied to displace the electron beam horizontally to bypass the crystal mirror and to match the delay of $\mathrm{x}$-ray pulse by crystal. The details of configuration and properties are investigated in the following. Since a high peak power FEL amplifier is pursued, only high peak current electron beam is considered in this paper.

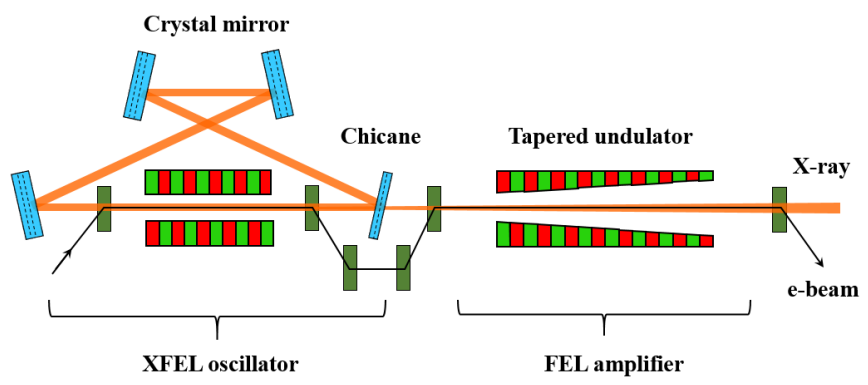

FIG. 1. The schematic of XFELO seeded FEL. 


\section{A. The drawbacks of cavity tuning}

A well-known drawback of this FEL oscillator-amplifier configuration is that due to the electron beam large energy spread after interactions with intense $\mathrm{x}$-ray electromagnetic field in the oscillator large amount of electrons may drop out of the "ponderomotive bucket," which will degrade the tapered FEL efficiency. There are multiple methods proposed for solving this problem, such as using self-modelocked oscillator-amplifier FEL configuration [35], and replacing undulator in oscillator by optical klystron [36]. Other simpler approaches, which manage to eliminate the beam energy spread growth in oscillator while avoiding the requirements of additional components, would be to generate faint $\mathrm{x}$-ray power inside the cavity so long as it dominates over the spontaneous radiation in the subsequent FEL amplifier. For example, an XFELO output with few MW peak power is sufficient as a signal to overwhelm the shot noise for a typical $3 \mathrm{kA}$ peak current electron beam.

To control the XFELO radiation intensity at a special level, the straightforward options are fine tuning of oscillator parameters, e.g., by increasing the output coupling or by detuning the cavity length. In the long wavelength region, this methods are plausible as discussed in [37]. However, for XFELO which uses crystal as cavity mirrors, the effects of crystal reflectivity and cavity length detuning are not clear yet and should be investigated. Using the same electron bunch parameters and cavity configuration as in Sec. III, three-dimensional simulations are carried out through a combination of GENESIS [38] and OPC [39] for crystal reflectivity, while a fast one-dimensional theoretical method is employed for cavity detune [40]. According to these results, however, aforementioned two approaches are proved to be infeasible.

The XFELO output peak power at various output mirror reflectivity is shown in Fig. 2. When the cavity output coupling is large, the small FEL gain cannot overcome round trip net loss, thus the x-ray power remains shot noise

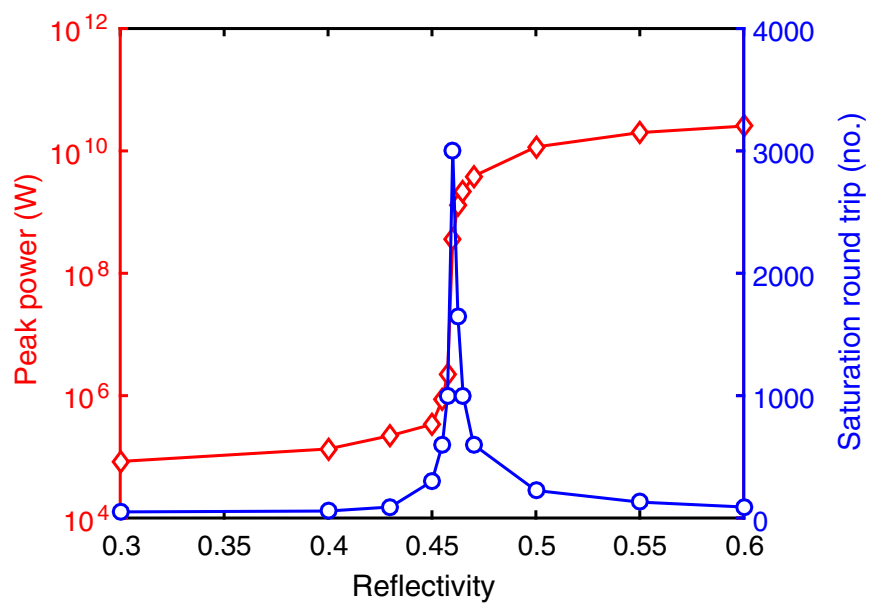

FIG. 2. The XFELO output peak power and saturation round trip number as a function of cavity output mirror reflectivity.

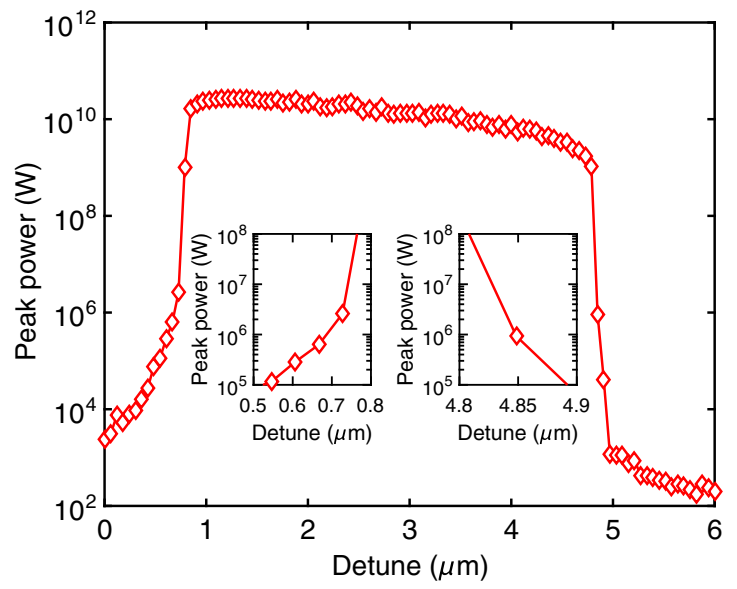

FIG. 3. The XFELO output peak power at different cavity detune.

level. As the oscillator feedback rises, Eq. (1) is satisfied and XFELO output increases rapidly to tens of GW. The steep curve indicates that generating stable MW level x-ray seed signal by changing cavity output coupling is unpractical, since it is hard to operate an XFELO at an unstable status. In addition, changing mirrors reflectivity finely by varying crystal thickness is challenging in practical. The round trips number required for XFELO saturation is also plotted in Fig. 2, which shows that much more passes are required to reach an equilibrium status around the critical output mirror reflectivity.

The influence of cavity detune on output x-ray power is presented in Fig. 3. According to [30,32], the symmetry Bragg reflection of diamond crystal $\left(\begin{array}{lll}1 & 1 & 1\end{array}\right)$ atomic planes with $1.09 \mu \mathrm{m}$ extinction length would lead to nearly $1.09 \mu \mathrm{m}$ delay in the radiation, which is consistent with the cavity mirror detuning required indicated in Fig. 3. According to the subfigures, cavity length control system with submicrometer resolution is required in order to obtain MW level output peak power. Due to the dramatically growth and decline of output peak power when XFELO deviates from the optimum synchronization, the cavity mirrors position jitter might lead to large output power fluctuation and undermines the following tapered FEL amplifier, which means obtaining faint $x$-ray inside XFELO by finely adjusting cavity length is also infeasible.

\section{B. The chirped-beam XFELO}

According to the previous results and discussions, an alternative strategy would be using twin bunch lasing [41] or recently developed fresh-slice technique [42], which however require relatively complicated machine setup and additional accelerator hardware components. In this paper, a simpler chirped-beam XFELO scheme is proposed for preserving energy spread of head part of electron beam while getting enough FEL gain from the tail. The lasing is localized at electron beam tail by arranging the chirp so that 


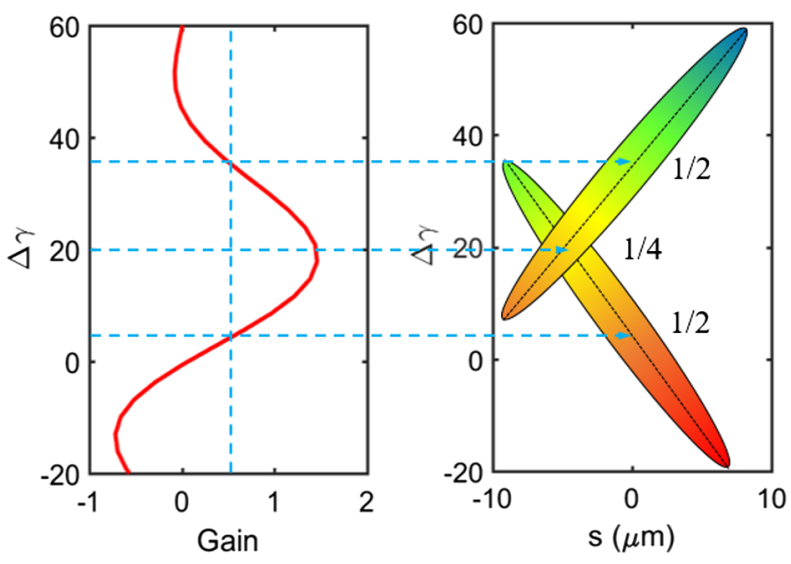

FIG. 4. The schematic of $3 \mathrm{kA}$ peak current XFELO single pass gain (red solid line) and optimized chirped-beam. Assuming that $50 \%$ gain is necessary, and the $1 / 4$ bunch tail point corresponds to the maximum single pass gain, while the central point relates to $50 \%$ single pass gain. Thus only bunch tail gets enough gain to compensate the round trip net loss, while bunch head does not lase significantly inside the XFELO.

the gain at the middle point compensates round trip loss and output coupling. In order to make full use of electron bunch, the gain at $1 / 4$ point is maximum. Schematic for optimization of a $3 \mathrm{kA}$ peak current chirped-beam for XFELO is demonstrated in Fig. 4. Assuming that single pass gain of $50 \%$ is needed for compensating round trip loss and output coupling. In order to guarantee only the bunch tail lases in XFELO, the electron energy of the beam center is set corresponding to $50 \%$ single pass gain, while the gain of $1 / 4$ point at the bunch tail is maximum, i.e., the electron beam chirp is

$$
\alpha=\frac{\Delta \gamma / \gamma_{0}}{\Delta l / L_{b}}=4 \frac{\gamma_{1 / 2}-\gamma_{1 / 4}}{\gamma_{0}},
$$

where $\gamma_{0}$ is the resonant electron energy measured in rest mass units, $L_{b}$ is the length of electron beam and $\Delta l$ is the length deviation corresponding to $\Delta \gamma$ energy detune, and $\gamma_{1 / 4}, \gamma_{1 / 2}$ represent the electron energy at $1 / 4$ and $1 / 2$ points. Note that an electron beam with both positive chirp and negative chirp can be used for the XFELO seeded FEL amplifier. The effect of linear chirp on FEL gain is equivalent to the linear tapered undulator, and thus the beam energy chirp could be compensated with the linear undulator taper and thus optimize the following amplification.

\section{Tapered FEL amplifier}

Tapered undulator technique is well-known for the capability of maintain resonant condition after FEL saturation, thus improving the energy extraction efficiency. In practice, however, it is hard to generate $\mathrm{x}$-ray peak power as high as estimated by simulations. As far as we know, one of the main reasons is lack of suitable seed radiation from previous undulators. On the one hand, SASE starts from electron initial random density fluctuations, and possess poorly longitudinal coherence, thus unstable generation of parasitic radiation with different frequency from the signal would occur [18], which causes the degeneration of tapered amplifier performance. On the other hand, although selfseeding scheme is able to generate $\mathrm{x}$-ray pulses with narrow bandwidth, nearly twice as much as undulator periods are required comparing to SASE, and even more undulators might be necessary for further improving the pulse energy by tapering technique. In addition, selecting a coherent seed from broad bandwidth SASE by the narrow acceptance of Bragg crystal diffraction leads to large shot-to-shot self-seeding pulse energy fluctuations, and matching the tapered undulators configurations for each individual shot is impossible. Furthermore, the relatively small seed signal after self-seeding, which means small "ponderomotive bucket" height, would cause portions of energy spread degraded electron beam escaping from bucket and reduce the efficiency of tapered FEL amplifier. For the chirpedbeam XFELO seeded FEL proposed in this paper, a fully coherent, high peak power stable $\mathrm{X}$-ray seed radiation from XFELO is benefit for the following tapered amplifier. And the large energy spread tail electrons are replaced by fresh parts of electron beam thus ensure the significant FEL gain inside following amplifier.

\section{TIME-DEPENDENT SIMULATION}

To illustrate the main principles of XFELO seeded FEL, the main parameters of SCLF are employed. SCLF is the first hard X-ray FEL facility in China which is now under construction. The major parameters of electron beam and undulator are summarized in Table. I. Taking advantage of superconducting accelerator technique and lower thermal emittance photocathodes, SCLF is capable of delivering nearly $1 \mathrm{MHz}$ relativistic electron bunches of FEL quality. The combination of high brightness electron beam and $200 \mathrm{~m}$ length variable gap undulators are suitable for XFELO seeded FEL amplifier. The simulations are

TABLE I. The main parameters of SCLF.

\begin{tabular}{lcc}
\hline \hline Parameter & Value & Unit \\
\hline Beam energy & 8 & $\mathrm{GeV}$ \\
Slice relative energy spread & 0.01 & $\%$ \\
Normalized emittance & 0.4 & $\mu \mathrm{m}-\mathrm{rad}$ \\
Repetition rate & 1 & $\mathrm{MHz}$ \\
Peak current & 3 & $\mathrm{kA}$ \\
Bunch charge & 100 & $\mathrm{pC}$ \\
Undulator period & 26 & $\mathrm{~mm}$ \\
Undulator mean beta function & 13 & $\mathrm{~m}$ \\
XFELO single pass gain & 1.5 & \\
Total undulator length & 200 & $\mathrm{~m}$ \\
\hline \hline
\end{tabular}


specially carried out at $5 \mathrm{keV}$ x-ray photons, since the production of high peak power and short temporal duration FEL radiation at that wavelength is of great interests for single-particle imaging and coherent diffraction imaging experiments.

For shortening tapering FEL gain length, high intensity $3 \mathrm{kA}$ flat-top current electron beam with $100 \mathrm{pC}$ bunch charge is used. The short electron bunch temporal duration corresponds to a broad spectral bandwidth (especially for XFELO seeded FEL in which only half of electron beam is lasing), and in order to avoid excessive cavity loss due to crystal mirror narrow bandwidth acceptance [32], the (1 11 1) atom plane of diamond crystal with incident angle $\theta=36.9^{\circ}$ is chosen to obtain a $324 \mathrm{meV}$ Darwin bandwidth. A $300 \mathrm{~m}$ round trip length optical cavity contains one module of undulator with 200 periods is used for XFELO to match the electron beam repetition rate and provide sufficient 1.5 single pass gain. The $150 \mathrm{~m}$ large distance between two cavity mirrors would leads to the requirement of $\sim 10 \mathrm{nrad}$ high resolution alignment, which might be achieved by the technique developed in [43]

The performances of XFELO seeded FEL are simulated by the combination of GENESIS and OPC, and the results are shown in Fig. 5, with the top row displays XFELO gain and output, while bottom row plots tapered FEL gain and output. The evolution of XFELO radiation pulse energy is shown in (a), which undergoes an exponential growth as expected and generates $206 \mu \mathrm{J}$ x-ray in the saturation regime. The electron beam chirp is first calculated by Eq. (2) and then optimized to be $\alpha=6.1 \times 10^{-3}$ through simulation. With $10 \mu \mathrm{m}$ electron bunch length, only the tail electrons contributes to FEL gain and induced large energy spread as illustrated in (b). The length of output x-ray pulse is $4.25 \mu \mathrm{m}$ (FWHM) which is nearly half of electron beam duration. The $\mathrm{x}$-ray beam broaden in temporal domain is mainly due to the spectral purification of crystal mirror Bragg diffraction, which also leads to $212.5 \mathrm{meV}$ (FWHM) output bandwidth.

As aforementioned, a chicane bypass is adjusted properly for delaying the bunch and let head electrons overlap with the seed radiation as well as to wash out the XFELOinduced microbunching. Quadratic tapering model is chosen and carefully optimized to obtain maximum pulse energy for subsequent nearly $180 \mathrm{~m}$ length tapered FEL amplifier. The second row of Fig. 5 plots the FEL gain and output pulse in tapered amplifier. The pulse energy displays a typical linear growth with undulator length increases. Simulation shows that the new scheme is able to generate $7.9 \mathrm{~mJ}$ pulse energy with $0.92 \mathrm{TW}$ peak power. The undulator magnetic intensity is optimized for electron beam slices corresponding to maximum seed signal from XFELO, thus the output $\mathrm{x}$-ray temporal duration is $14 \mathrm{fs}$ (FWHM), which is shorter than XFELO output radiation. The chirped electron beam leads the spectral bandwidth broaden to $586 \mathrm{meV}$ (FWHM) which corresponds to a time-bandwidth product of 2 , larger than the value of
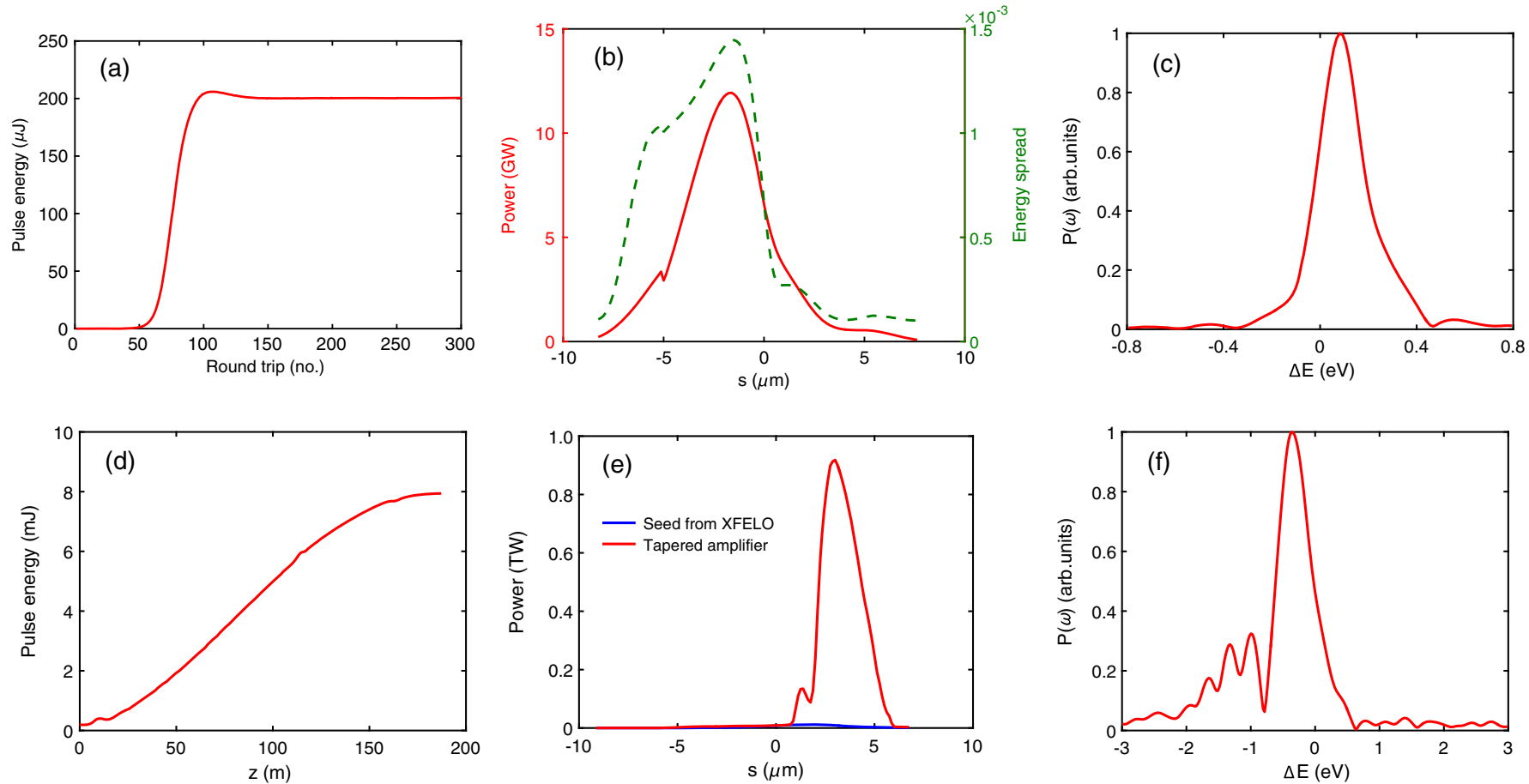

FIG. 5. The performance of $3 \mathrm{kA}$ peak current flat-top electron beam for XFELO with $18 \%$ crystal mirror output coupling. The top row shows (a) output pulse energy gain, (b) power profile (red solid line) and beam energy spread (green dashed line), (c) spectrum for XFELO, while the bottom row plots the FEL amplifier energy gain (d), seed (blue line) and output power profile (red line) (e), and spectrum at the end of undulators (f). 
Fourier transform limit (0.44) for Gaussian pulse profile. The time-dependent results proved that it is feasible to generate high brightness $\mathrm{x}$-ray pulses with the chirped beam scheme.

\section{START-TO-END SIMULATION OF SCLF}

The real performances of XFELO seeded FEL are evaluated by start-to-end simulations of SCLF. The electron beam dynamics simulation in the photon-injector is carried out by ASTRA [44] with space charge effects taken into account. ELEGANT [45] is then used for simulation in the reminder of Linac. For pursuing shorter gain length of SASE FEL, the electrons are usually accelerated off-crest in the superconducting Linac and possess large energy chirp, then the bunch is compressed longitudinally inside a chicane compressor to obtain high peak current. In order to achieve high efficient high-gain FELs, the residual energy chirp compensation is of paramount importance and several techniques are available for reaching this goal, i.e., off-crest dechirping and passively dechirping through linac cavity wakefield. For SCLF using low-frequency superconducting technology, however, the wakefield is too weak to remove the residual chirp, and actively dechirping by running the beam off-crest in a linac is an inefficient and costly option. Thus SCLF takes advantages of an inexpensive $12 \mathrm{~m}$ length corrugated pipe as a beam dechirper [46-48].

In this paper, however, we explore the application of introduced chirp in XFELO seeded FEL operation without additional chirp removal devices. The phase space electrons distribution (a) and the corresponding current profile (b) at the end of bunch compressor are presented in Fig. 6. In the case of a given chirp, using the strategies demonstrated in Fig. 4, the cavity mirror reflectivity could be adjusted for the fulfillment of Eq. (2). With the FEL gain of small current between two horns being ignored, the natural "double-horn" of electron beam is proved to be quite suitable for our scheme: the head horn is set to be outside the gain bandwidth and well-preserved for FEL amplifier; while the tail horn corresponding to maximum single pass gain is lasing inside the XFELO, and combine with the large distance $14 \mu \mathrm{m}$ between the two horns would leads to relative small optimized energy chirp. In the simulation, the output coupling is adjusted to be $33 \%$ for suitable gain bandwidth, and the residual chirp $\alpha=-1.3 \times 10^{-3}$ in SCLF is large enough to localize resonant FEL interaction at tail. In addition, the large energy spread of the "double horn" current is not as harmful to XFELO seeded FEL as self-seeding scheme, since neither the high pear current XFELO, which works in the small signal regime with fairly large gain bandwidth, nor the tapered FEL amplifier with high intensity seed signal is sensitive to electron beam energy spread.

The simulations were conducted with the same XFELO configuration in Sec. III for $5 \mathrm{keV}$ photons. The XFELO pulse energy growth and output are shown in the upper row of Fig. 7, which demonstrates that the tail horn produces a nearly $190 \mu \mathrm{J}, 14.5 \mathrm{GW}$ XFELO output, with $0.3 \mathrm{eV}$ (FWHM) spectral bandwidth. The energy spread increasing is bounded to the bunch tail as indicated in subfigure (b). The high peak power density of XFELO is nearly $190 \mathrm{GW} / \mathrm{cm}^{2}$, which might be is a challenge for cavity crystal mirror thermal loading tolerance as discussed in [49]. The FEL growth and output tapered of FEL amplifier are presented in the bottom row of Fig. 7. The XFELO output is amplified to $3.8 \mathrm{~mJ}, 0.55 \mathrm{TW}$, with $0.66 \mathrm{eV}$ (FWHM) spectral bandwidth and 5.5 fs (FWHM) temporal duration. This corresponds to a time-bandwidth product of 0.88 , which is twice the value of Fourier transform limit (0.44) for Gaussian pulse profile. Due to the relatively small bunch energy chirp as well as short radiation duration, the output pulse is nearly fully longitudinal coherent. The XFELO seeded FEL is able to generate $5 \mathrm{keV}$ x-ray photons with $3.35 \times 10^{35}$ (photons/ $\mathrm{s} / \mathrm{mm}^{2} / \mathrm{mrad}^{2} / 0.1 \% \mathrm{BW}$ ) peak brilliance, which is two order of magnitude larger than SASE FEL brilliance $1.1 \times 10^{33}$ (photons $/ \mathrm{s} / \mathrm{mm}^{2} / \mathrm{mrad}^{2} / 0.1 \% \mathrm{BW}$ ) produced by the same electron and undulator parameters.

To eliminate the influence of phase mismatching of tapering technique, the undulators without break sections are employed in the previous simulations. For revealing the real performance of tapered FEL amplifier in SCLF, the simulation is conducted with nearly $1 \mathrm{~m}$ break between two segments of undulators. The normalized field amplitudes of

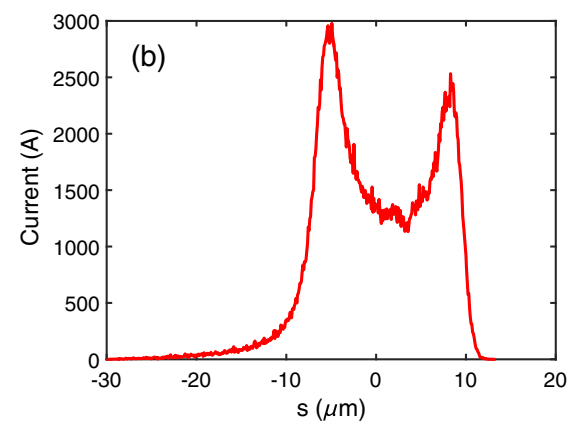

FIG. 6. The electrons distribution in phase space (a) and the corresponding current profile (b). 

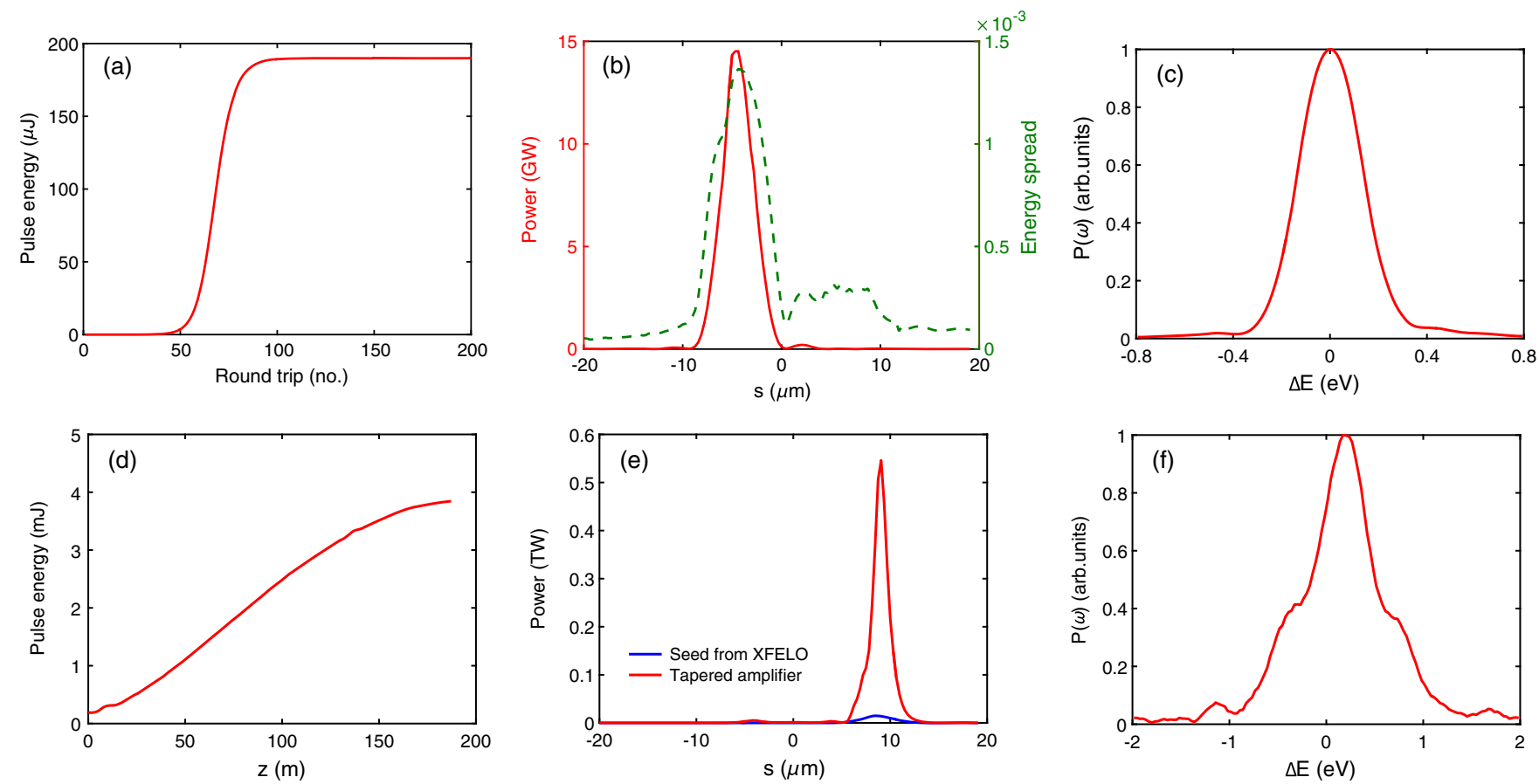

FIG. 7. The start-to-end simulation results for XFELO with the 33\% crystal mirror output coupling is shown in the top row of panel: (a) output pulse energy growth at different round trip, (b) power profile (red solid line) and beam energy spread (green dashed line), (c) spectrum for XFELO output. The bottom row plots the out pulse energy gin in the tapered FEL (d), seeding from XFELO (blue line) and output power profile (red line) (e), and the spectrum at the end of undulators (f).

36 segments of undulators and intensity of the phase shifter are optimized by genetic algorithm [50]. Due to the constant normalized undulator field intensity in one undulator segments, a portion of electrons would leak out the "ponderomotive bucket" and causes the pulse energy reduce to $3.1 \mathrm{~mJ}$ with $0.36 \mathrm{TW}$ peak power.

\section{OUTPUT ENERGY STABILITY}

Benefiting from the high intensity stable seed from XFELO, the output X-ray beam is expected to be of highly robustness. One of the key factors that influence the operation stability is the FEL gain fluctuation, which is mainly due to electron beam quality variation, e.g., bunch energy jitter. To illustrate the stability of XFELO seeded FEL in real machine, 100 start-to-end runs are carried out at each rms value of electron beam energy jitter. The results of XFELO (a) and tapered amplifier (b) with energy fluctuation rms equals to $10^{-4}$ are shown in Fig. 8. The gray lines refer to 100 separate runs and the red line is the average value of them. The results are summarized in Fig. 8(c), which indicates that the output pulse energy fluctuation increases as electron bunch energy jitter rises, and with the typical $10^{-4} \mathrm{rms}$ beam energy jitter in SCLF, XFELO seeded FEL is able to generate high brightness $\mathrm{X}$-ray pulse with pulse energy rms as low as $3 \%$. Note that the final output is more robust to bunch energy jitter than
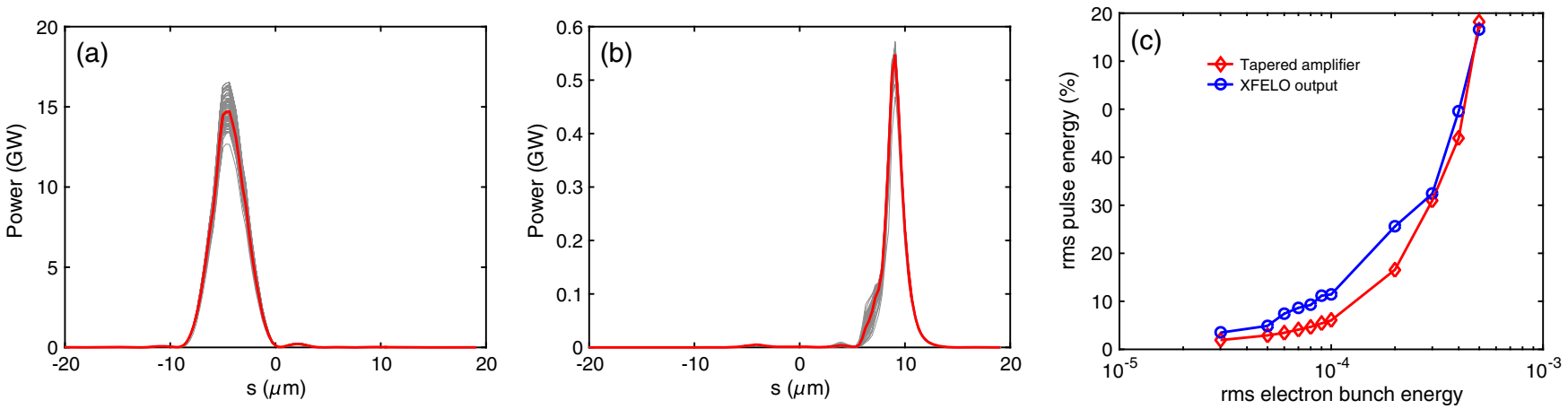

FIG. 8. The output radiation of (a) XFELO and (b) tapered FEL amplifier. The gray lines refer to 100 runs with $10^{-4}$ rms beam energy jitter, and red line is the average value. Subfigure (c) shows the rms of output energy with different rms beam energy jitter. 
XFELO, further analysis reveals that this is due to the relatively broader gain bandwidth of tapered FEL amplifier in respect XFELO in our simulations.

\section{CONCLUSION}

The chirped-beam FEL oscillator-amplifier is a novel scheme for generating ultra-high brightness, fully coherent x-ray beam. The XFELO output provides a high intensity, stable seed signal, which is a favorable "bucket" in the phase space to trap electrons and to maintain consistent energy extraction in tapered FEL amplifier. The chirped beam is optimized for ensuring the bunch tail lases effectively in XFELO while the bunch head is preserved for following tapered FEL amplifier. It is demonstrated that with optimized parameters, the new scheme is able to generate a few $\mathrm{mJ}$, TW level fully coherent $5 \mathrm{keV} \mathrm{x}$-ray radiation.

Taking advantage of the natural remnant energy chirp after chicane compressor, a start-to-end simulation is conducted using the basic parameters in SCLF. The "double horn" current is proved to be favorable to XFELO seeded FEL operation. Simulation results indicate that the new scheme is capable of generating $3.8 \mathrm{~mJ}$ pulse energy, $0.55 \mathrm{TW}$ peak power, $5 \mathrm{keV}$ x-ray photon with peak brilliance two order of magnitude larger than SASE FEL generated in SCLF. In addition, XFELO seeded FEL output is quite stable, the output pulse energy rms fluctuation is as small as 3\% for a typical $10^{-4}$ rms electron beam energy jitter. The ideal x-ray light source is expected to be valuable for some special experiments, i.e., x-ray single-shot diffraction imaging and coherent scattering techniques, which require high spectral brightness radiation. Note that the potential of this new scheme might be further exploited with fully optimization of electron beam chirp, XFELO output coupling and the normalized field intensity of tapered undulators. The following work would be to analyze the influence of bandwidth of seeding radiation on its performances as well as the gain bandwidth of tapered FEL amplifier.

\section{ACKNOWLEDGMENTS}

The author would like to thank B. Liu, D. Wang and Z. Zhao for helpful discussions on SCLF project; T. Liu for enthusiastic discussions on FEL tapering physics. This work was partially supported by the National Natural Science Foundation of China (11775293), the National Key Research and Development Program of China (2016YFA0401900), the Young Elite Scientist Sponsorship Program by CAST (2015QNRC001) and Ten Thousand Talent Program.

[1] A. M. Kondratenko and E. L. Saldin, Generation of coherent radiation by a relativistic electron beam in an ondulator, Part. Accel. 10, 207 (1980).
[2] R. Bonifacio, C. Pellegrini, and L. Narducci, Collective instabilities and high-gain regime in a free electron laser, Opt. Commun. 50, 373 (1984).

[3] P. Emma, R. Akre, J. Arthur, R. Bionta, C. Bostedt, J. Bozek, A. Brachmann, P. Bucksbaum, R. Coffee, F.-J. Decker et al., First lasing and operation of an ångstromwavelength free-electron laser, Nat. Photonics 4, 641 (2010).

[4] D. Pile, X-rays: First light from SACLA, Nat. Photonics 5, 456 (2011).

[5] H.-S. Kang, K.-W. Kim, and I. S. Ko, in Proceedings of 4th International Particle Accelerator Conference (JACOW, Shanghai, China, 2013), p. 2074.

[6] M. Altarelli, R. Brinkmann, M. Chergui, W. Decking, B. Dobson, S. Düsterer, G. Grübel, W. Graeff, H. Graafsma, J. Hajdu et al., The European X-ray free-electron laser, Technical Design Report, DESY 97, 1 (2006).

[7] C. Bostedt, S. Boutet, D. M. Fritz, Z. Huang, H. J. Lee, H. T. Lemke, A. Robert, W. F. Schlotter, J. J. Turner, and G. J. Williams, Linac Coherent Light Source: The first five years, Rev. Mod. Phys. 88, 015007 (2016).

[8] G. Geloni, V. Kocharyan, and E. Saldin, A novel selfseeding scheme for hard X-ray FELs, J. Mod. Opt. 58, 1391 (2011).

[9] J. Amann, W. Berg, V. Blank, F.-J. Decker, Y. Ding, P. Emma, Y. Feng, J. Frisch, D. Fritz, J. Hastings et al., Demonstration of self-seeding in a hard-X-ray freeelectron laser, Nat. Photonics 6, 693 (2012).

[10] D. Xiang, Y. Ding, Z. Huang, and H. Deng, Purified selfamplified spontaneous emission free-electron lasers with slippage-boosted filtering, Phys. Rev. ST Accel. Beams 16, 010703 (2013).

[11] B. W. J. McNeil, N. R. Thompson, and D. J. Dunning, Transform-Limited X-Ray Pulse Generation from a High-Brightness Self-Amplified Spontaneous-Emission Free-Electron Laser, Phys. Rev. Lett. 110, 134802 (2013).

[12] J. Wu, A. Marinelli, and C. Pellegrini, in Proceedings of the 34th International Free Electron Laser Conference (JACOW, Nara, Japan, 2012), p. 237.

[13] L. H. Yu, Generation of intense uv radiation by subharmonically seeded single-pass free-electron lasers, Phys. Rev. A 44, 5178 (1991).

[14] G. Stupakov, Using the Beam-Echo Effect for Generation of Short-Wavelength Radiation, Phys. Rev. Lett. 102, 074801 (2009).

[15] H. Deng and C. Feng, Using Off-Resonance Laser Modulation for Beam-Energy-Spread Cooling in Generation of Short-Wavelength Radiation, Phys. Rev. Lett. 111, 084801 (2013).

[16] H. N. Chapman, P. Fromme, A. Barty, T. A. White, R. A. Kirian, A. Aquila, M. S. Hunter, J. Schulz, D. P. DePonte, U. Weierstall et al., Femtosecond X-ray protein nanocrystallography, Nature (London) 470, 73 (2011).

[17] M. M. Seibert, T. Ekeberg, F. R. Maia, M. Svenda, J. Andreasson, O. Jönsson, D. Odić, B. Iwan, A. Rocker, D. Westphal et al., Single mimivirus particles intercepted and imaged with an X-ray laser, Nature (London) 470, 78 (2011).

[18] N. Kroll, P. Morton, and M. Rosenbluth, Free-electron lasers with variable parameter wigglers, IEEE J. Quantum Electron. 17, 1436 (1981). 
[19] E. A. Schneidmiller and M. Yurkov, Optimization of a high efficiency free electron laser amplifier, Phys. Rev. ST Accel. Beams 18, 030705 (2015).

[20] Y. Jiao, J. Wu, Y. Cai, A. Chao, W. Fawley, J. Frisch, Z. Huang, H.-D. Nuhn, C. Pellegrini, and S. Reiche, Modeling and multidimensional optimization of a tapered free electron laser, Phys. Rev. ST Accel. Beams 15, 050704 (2012).

[21] T. J. Orzechowski, B. Anderson, J. C. Clark, W. M. Fawley, A. C. Paul, D. Prosnitz, E. T. Scharlemann, S. M. Yarema, D. B. Hopkins, A. M. Sessler et al., High-Efficiency Extraction of Microwave Radiation from a TaperedWiggler Free-Electron Laser, Phys. Rev. Lett. 57, 2172 (1986).

[22] X. J. Wang, H. P. Freund, D. Harder, W. H. Miner Jr, J. B. Murphy, H. Qian, Y. Shen, and X. Yang, Efficiency and Spectrum Enhancement in a Tapered Free-Electron Laser Amplifier, Phys. Rev. Lett. 103, 154801 (2009).

[23] Z. Huang and G. Stupakov, Free electron lasers with slowly varying beam and undulator parameters, Phys. Rev. ST Accel. Beams 8, 040702 (2005).

[24] E. L. Saldin, E. A. Schneidmiller, and M. V. Yurkov, Selfamplified spontaneous emission FEL with energy-chirped electron beam and its application for generation of attosecond x-ray pulses, Phys. Rev. ST Accel. Beams 9, 050702 (2006).

[25] H. Deng and C. Feng, in Proceedings of the 4th International Particle Accelerator Conference (JACOW, Shanghai, China, 2013), p. 1214.

[26] K.-J. Kim, Y. Shvydko, and S. Reiche, A Proposal for an X-Ray Free-Electron Laser Oscillator with an EnergyRecovery Linac, Phys. Rev. Lett. 100, 244802 (2008).

[27] J. Dai, H. Deng, and Z. Dai, Proposal for an X-Ray Free Electron Laser Oscillator with Intermediate Energy Electron Beam, Phys. Rev. Lett. 108, 034802 (2012).

[28] Z. Zhu, Z. Zhao, D. Wang, Z. Liu, R. Li, L. Yin, and Z. Yang, in Proceedings of the FEL2017, Santa Fe, NM, USA (2017), p. 20 (to be published).

[29] Y. V. ShvydKo, S. Stoupin, A. Cunsolo, A. H. Said, and X. Huang, High-reflectivity high-resolution X-ray crystal optics with diamonds, Nat. Phys. 6, 196 (2010).

[30] R. Lindberg, K.-J. Kim, Y. Shvydko, and W. Fawley, Performance of the X-ray free-electron laser oscillator with crystal cavity, Phys. Rev. ST Accel. Beams 14, 010701 (2011).

[31] M. Borland, G. Decker, A. Nassiri, and A. White, in Proceedings of Particle Accelerator Conference 07 (IEEE, Albuquerque, NM USA, 2007), p. 1121.

[32] Y. Shvydko and R. Lindberg, Spatiotemporal response of crystals in x-ray Bragg diffraction, Phys. Rev. ST Accel. Beams 15, 100702 (2012).

[33] P. Pierini, M. Bertucci, A. Bosotti, J. Chen, C. Maiano, P. Michelato, L. Monaco, M. Moretti, C. Pagani, R. Paparella et al., Fabrication and vertical test experience of the European X-ray Free Electron Laser 3.9 GHz superconducting cavities, Phys. Rev. Accel. Beams 20, 042006 (2017).

[34] A. Burrill, K. Davis, D. Gonnella, A. Grassellino, O. Melnychuk, A. Palczewski, M. Ross, and L. Zhao, in
Proceedings of 8th International Particle Accelerator Conference (JACOW, Copenhagen, Denmark, 2017), p. 1152 .

[35] D. J. Bamford and D. A. Deacon, Self-mode-locked oscillator-amplifier FEL configuration, Nucl. Instrum. Methods Phys. Res., Sect. A 304, 667 (1991).

[36] G. Dattoli, L. Giannessi, and P. Ottaviani, in Free Electron Lasers 2002 (Elsevier, New York, 2003), p. 26.

[37] G. Dattoli, L. Mezi, A. Renieri, and A. Torre, Freeelectron laser saturated regime, cavity modulation, and harmonic generation, IEEE J. Quantum Electron. 34, 1782 (1998).

[38] S. Reiche, GENESIS 1.3: a fully 3D time-dependent FEL simulation code, Nucl. Instrum. Methods Phys. Res., Sect. A 429, 243 (1999).

[39] P. J. M. van der Slot, H. P. Freund, W. H. Miner Jr, S. V. Benson, M. Shinn, and K.-J. Boller, Time-Dependent, Three-Dimensional Simulation of Free-Electron-Laser Oscillators, Phys. Rev. Lett. 102, 244802 (2009).

[40] K. Li, M. Song, and H. Deng, Simplified model for fast optimization of a free-electron laser oscillator, Phys. Rev. Accel. Beams 20, 030702 (2017).

[41] W. Qin, K.-J. Kim, R. Lindberg, and J. Wu, in Proceedings of 38th International Free Electron Laser Conference (JACOW, Santa Fe, NM USA, 2017).

[42] A. A. Lutman, T. J. Maxwell, J. P. MacArthur, M. W. Guetg, N. Berrah, R. N. Coffee, Y. Ding, Z. Huang, A. Marinelli, S. Moeller et al., Fresh-slice multicolour X-ray free-electron lasers, Nat. Photonics 10, 745 (2016).

[43] D. Shu, W.-K. Lee, W. Liu, G. E. Ice, Y. Shvydko, and K.-J. Kim, Development and applications of a twodimensional tip-tilting stage system with nanoradian-level positioning resolution, Nucl. Instrum. Methods Phys. Res., Sect. A 649, 114 (2011).

[44] K. Flöttmann et al., ASTRA: A Space Charge Tracking Algorithm, http://www.desy.de/ mpyflo/ Astra_dokumentation/.

[45] M. Borland, Report No. LS-287, 2000.

[46] K. Bane and G. Stupakov, Corrugated pipe as a beam dechirper, Nucl. Instrum. Methods Phys. Res., Sect. A 690, 106 (2012).

[47] S. Antipov, C. Jing, M. Fedurin, W. Gai, A. Kanareykin, K. Kusche, P. Schoessow, V. Yakimenko, and A. Zholents, Experimental Observation of Energy Modulation in Electron Beams Passing through Terahertz Dielectric Wakefield Structures, Phys. Rev. Lett. 108, 144801 (2012).

[48] H. Deng, M. Zhang, C. Feng, T. Zhang, X. Wang, T. Lan, L. Feng, W. Zhang, X. Liu, H. Yao et al., Experimental Demonstration of Longitudinal Beam Phase-Space Linearizer in a Free-Electron Laser Facility by Corrugated Structures, Phys. Rev. Lett. 113, 254802 (2014).

[49] M.-Q. Song, Q.-M. Zhang, Y.-H. Guo, K. Li, and H.-X. Deng, Numerical modeling of thermal loading of diamond crystal in X-ray FEL oscillators, Chin. Phys. C 40, 048101 (2016).

[50] K. Deb, A. Pratap, S. Agarwal, and T. Meyarivan, A fast and elitist multiobjective genetic algorithm: NSGA-II, IEEE Trans. Evol. Comput. 6, 182 (2002). 